\title{
Les aspects sociaux d'une invasion biologique en forêt domaniale de Compiègne : la construction sociale de Prunus serotina
}

\author{
Aurélie Javelle $^{\mathrm{a}}$, Bernard Kalaora ${ }^{\mathrm{b}}$, Guillaume Decocq ${ }^{\mathrm{c}}$ \\ a Doctorante en ethnologie, Université Rennes 1, ECOBIO, UMR 6553, Campus Beaulieu, 35042 Rennes cedex, France \\ b Sociologue, LAIOS, EHESS, 54 bd Raspail, 75006, Paris, France \\ c Professeur de botanique, Université de Picardie Jules Verne, 1 rue des Louvels, 80037 Amiens cedex, France
}

\section{Mots-clés :}

invasion biologique;

Prunus serotina; perception;

biodiversité ; forêt

\section{Keywords:}

biological invasion;

Prunus serotina;

perception;

biodiversity;

forest

\begin{abstract}
Résumé - Le cerisier tardif (Prunus serotina Ehrh.) est un arbre d'origine nord-américaine qui envahit les forêts tempérées européennes. En France, la forêt de Compiègne, domestiquée par des aménagements sylvicoles et récréatifs dont l'origine remonte au Second Empire, est la plus touchée. Prunus serotina vient troubler l'ordonnancement sylvicole et paysager ainsi que l'exploitabilité des forêts, invalidant de ce fait la maîtrise des gestionnaires et des propriétaires privés ou publics. Les auteurs, une ethnologue, un sociologue et un écologue, tentent de saisir et de comprendre les processus de reconnaissance qui conduisent à l'identification de l'espèce par les différents usagers et acteurs de la forêt. Au-delà des objectifs théoriques, il s'agit de fournir une aide à la décision en vue d'établir des dispositifs d'alerte pour la prise en compte du phénomène « invasion biologique», inédit et mal connu.
\end{abstract}

Pour penser et revendiquer la forêt (plus largement, la nature), la constituer en cause légitime en vue de sa protection, nous disposons depuis le XIX' siècle d'une grammaire constituée et actualisable (Kalaora et Savoye, 1986). Le paysage forestier émerge au travers d'une ample activité sociale de configuration et de construction de motifs centrés sur le regard et la prééminence du bien-voir, en rapport avec l'activité picturale et littéraire. Cette activité suppose le tri des objets pertinents dans l'espace, le rapprochement des parties constituantes et repérées comme " plaisantes perspectives ", l'articulation et l'assemblage des «morceaux de nature » en une unité indissociable

Auteur correspondant :

A. Javelle, aurelie.javelle@univ-rennes1.fr qui fait paysage. La problématique des invasions biologiques, associée à l'émergence de l'environnement et à la question de la biodiversité, met à mal les schèmes paysagers reposant sur le paysagisme et l'art du pittoresque (Kalaora, 1993 ; Bertho-Lavenir, 1999) et ceux, pratiques, d'organisation matérielle et économique, représentant la forêt comme un peuplement sylvicole destiné tout à la fois à l'exploitation et la conservation. La biodiversité ne constitue pas encore une grammaire pouvant se substituer à la codification paysagère qui, elle, résulte d'un long apprentissage, à la fois discursif, esthétique et pratique. Un nouveau référentiel, que l'on peut qualifier d'environnemental et de biologique, s'ajoute au modèle classique de la forêt productive et paysagère (Theys et Kalaora, 1998). Ce rééquilibrage des fonctions, induit 
par la nécessité d'intégrer la biodiversité, n'est pas sans conséquence sur la pratique forestière, le rapport à l'espace, mais aussi aux savoirs que les forestiers vont être conduits à mobiliser pour faire face à ces nouveaux enjeux biologiques. Les invasions biologiques, dans cette perspective, constituent un exemple parlant d'un problème dont la définition relève de l'intégration de savoirs plus complexes que le savoir sylvicole et dont la gestion implique la mobilisation d'un public intéressé à la conservation de la forêt au sens large du terme (du vivant) et non seulement des paysages remarquables. La reconnaissance sociale d'un problème environnemental ne relève cependant pas exclusivement $d$ 'une science naturaliste ou écologique; elle suppose des relais et une mise en relation de partenaires avec qui discuter et négocier les nouveaux intérêts à représenter. Dans le cadre du programme INVABIO $\mathrm{II}^{1}$, le département de Botanique de l'Université de Picardie Jules Verne a pris comme objet d'étude et de réflexion l'invasion de la forêt domaniale de Compiègne par Prunus serotina, un arbre originaire d'Amérique du Nord. Ce projet interdisciplinaire ${ }^{2}$ regroupe écologues, géographes, mathématiciens, sociologues et gestionnaires pour mieux comprendre la dynamique invasive de cet arbre en système forestier tempéré. L'ethnologie a été sollicitée pour comprendre les conditions sociales du développement effectif de l'arbre allochtone en forêt de Compiègne, mais aussi sa perception, la manière dont les informations sur ce processus invasif ont circulé et se sont diffusées chez le public comme chez les professionnels de la filière forêt. Ce travail a permis de soulever des questions à propos de l'usage social de données des sciences de la nature concernant les invasions biologiques, ainsi que sur la perception d'un tel événement ayant lieu en forêt de Compiègne. Les données écologiques ont été confrontées aux réactions des acteurs locaux, afin d'illustrer les enjeux, les intérêts et les interférences de ceux-ci face à cette situation. L'enquête a été réalisée à partir d'entretiens semi-directifs et d'observations in situ, ainsi que d'analyses de documentation scientifique et de la presse, tous publics confondus.

\section{Le sujet de l'étude}

Prunus serotina, de Prunus (prunier : nom de l'arbre) et serotinus (tardif, automnal), est de la même famille que le merisier européen. Son nom vient du fait que sa floraison est très tardive par rapport aux autres espèces du

\footnotetext{
1 Programme de recherche lancé par le Comité interministériel de la recherche scientifique et technique.

2 «Dynamique invasive du cerisier tardif, Prunus serotina Ehrh., en système forestier tempéré : déterminants, mécanismes, impacts écologiques, économiques et socioanthropologiques ", programme INVABIO II dirigé par G. Decocq (2003-2006).
}

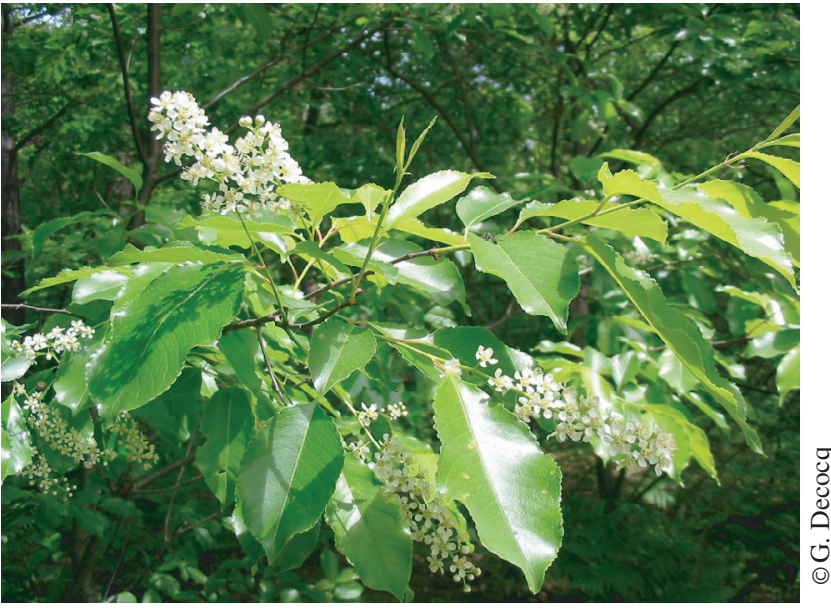

Photo. Prunus serotina.

genre (cf. photo), notamment Prunus padus (le cerisier à grappes), espèce autochtone avec laquelle il est souvent confondu. Prunus serotina est originaire d'une zone qui s'étend des Grands Lacs au golfe du Mexique (Hough et Forbes, 1943). Il a la particularité de drageonner et de rejeter de souche de façon très dynamique. Il se propage grâce à la dissémination de ses nombreuses graines, qui germent et forment un tapis de plantules capables de végéter plusieurs décennies en sous-bois. Dès qu'une arrivée de lumière se produit, par exemple après un chablis ou une coupe, sa croissance, beaucoup plus rapide que celle des essences indigènes, permet au Prunus serotina de conquérir l'espace. Il a donc toutes les qualités pour être un bon envahisseur biologique (Starfinger, 1997; Joly, 2000). C'est ainsi qu'aujourd'hui, il est présent sur près de $80 \%$ de la forêt. Telles sont, brièvement, les données botaniques qui expliquent en partie la situation actuelle.

\section{Origine de l'introduction}

On manque d'informations précises sur les origines de l'apparition de Prunus serotina en forêt de Compiègne. Le recoupement d'informations historiques par la " petite histoire » locale ${ }^{3}$ permet cependant d'échafauder une hypothèse. On raconte que c'est Napoléon III, fervent chasseur de faisans, qui aurait introduit l'arbre (lieu-dit «la Faisanderie »). En effet, ses fruits sont très appétents pour les oiseaux et il peut former un couvert végétal idéal puisqu'il peut être maintenu sous forme arbustive. Néanmoins, la consultation des archives indique des lacunes dans cette version. En effet, des documents

\footnotetext{
Personne, à Compiègne, ne parle spontanément de Prunus serotina. Face aux questions des personnes extérieures, la population «forestière » met en place une assise populaire à l'apparition de cet arbre à Compiègne, qui coïncide avec sa représentation de la forêt, même si la véracité n'en est nullement vérifiée.
} 
(Léré, 1819-1834, p. 147) décrivent précisément l'élevage de faisans et indiquent que trois hommes étaient employés pour fournir des œufs de fourmis récoltés en forêt. Nulle mention n'est faite d'un arbre destiné à nourrir ou à héberger les faisans, introduits dans un but cynégétique.

Si l'on continue la prospection, on découvre l'existence d'une école d'essais pour la culture des arbres exotiques dans un autre endroit de la forêt (lieu-dit «les Beaux Monts »). Une liste des espèces présentes y est dressée en 1855 (Graves, 1983, p. 224), mais dont est absent Prunus serotina. Enfin, un inventaire de 1827 (Léré, 1827-1839, p. 248) recensant les plantes du jardin du château royal de Compiègne, qui est enclavé dans la forêt, indique à plusieurs reprises Prunus virginiana avec un point d'interrogation. Sachant que ces deux Prunus sont très ressemblants, on peut opter pour l'hypothèse d'une introduction antérieure à Napoléon III dans un but esthétique pour suivre la mode des jardins à l'anglaise. Le parc touchant la forêt, Prunus serotina se serait progressivement échappé du premier pour coloniser la seconde. On observe le même cas de figure à Fontainebleau où cet arbre, présent dans les jardins de particuliers, s'échappe pour coloniser la forêt... L'histoire se répéterait-elle? On peut faire le lien entre l'histoire et l'actualité par cette même zone, entre parc et forêt, où Prunus serotina a fait parler de lui pour la première fois plus d'un siècle plus tard. C'est aussi dans ce secteur que l'individu le plus âgé (environ 150 ans) a été inventorié lors de nos prospections.

\section{La « découverte » de Prunus serotina}

Nous sommes à la fin des années 1960. Un agent forestier débute alors sa carrière à Compiègne. Il tente une régénération de hêtres en 1968 sur une parcelle à proximité du parc du château. C'est un échec. Il en découvre la raison, qui est la présence anormalement importante d'un arbre que tous ceux qui l'ont remarqué appellent «cerisier à grappes». En terminologie botanique, le cerisier à grappes correspond au Prunus padus, présent en forêt de Compiègne à l'état naturel. Cependant, si cet arbre était du Prunus padus, il pousserait en zone humide, ce qui n'est pas le cas. L'agent forestier constate qu'il s'installe extrêmement rapidement après les coupes de régénération ${ }^{4}$. Il se rend compte que l'espèce s'est établie sur près du tiers de la forêt. Face à la situation, il fait appel, en 1971, à un botaniste de l'université de Lille, afin d'identifier cet inconnu. Le prétendu cerisier à grappes se trouve en fait être du Prunus serotina, ou cerisier tardif. Le botaniste n'ajoutera aucun autre commentaire, ce

\footnotetext{
${ }^{4}$ Coupes d'arbres destinés à la vente, remplacés ensuite par d'autres à exploiter.
}

qu'il explique aujourd'hui en soulignant que la notion d'invasion biologique n'était alors pas répandue.

\section{Les aspects sylvicoles: des conditions favorables à l'explosion du Prunus serotina dans les années 1960-1980}

Les méthodes sylvicoles utilisées par l'Office national des forêts (ONF), doté de moyens importants au moment de sa création dans les années $1960^{5}$, peuvent expliquer le concours de circonstances à l'origine de l'explosion du Prunus serotina à Compiègne. D'une part, lorsque l'ONF plante alors une essence objectif ${ }^{6}$ dans une parcelle, le sol subit une préparation importante, très agressive. Les andains de rassemblement des souches peuvent encore se voir aujourd'hui. Or, comme beaucoup de plantes invasives, Prunus serotina aime les sols perturbés, qui lui fournissent les conditions idéales, très peu connues à l'époque, à son développement. Ensuite, les coupes à blanc, qui rentrent dans cette pratique sylvicole, favorisent également son développement en fournissant la lumière nécessaire aux plantules «dormantes » disséminées dans la forêt depuis son introduction au XIXe siècle. Dans le même sens, la dynamisation de la sylviculture du hêtre faisait également partie des objectifs de l'Office. Cela implique une futaie très lumineuse, donc favorable à la croissance de l'arbre invasif. Enfin, l'acide cyanhydrique présent dans l'écorce de Prunus serotina le protège des dents des grands animaux, en particulier du cerf et du chevreuil, qui se reportent alors sur ses « concurrents » que sont les essences objectifs.

\section{Les aspects sociaux : des actions échelonnées dans le temps}

Face à ces données, il s'agit à présent de se tourner vers les acteurs. Tout d'abord, considérons l'ONF, le premier concerné par le phénomène. L'agent forestier qui a découvert l'invasion s'est heurté à la résistance de l'arbre invasif, qui se multipliait après chaque tentative mécanique ou chimique pour l'éliminer. Une fois l'essence identifiée, il tente d'en savoir plus et recherche des informations, mais il se retrouve démuni : seul le Centre technique du bois et de l'ameublement (CTBA) lui fournit une

\footnotetext{
${ }^{5}$ Il s'agissait en effet de rattraper un retard de régénérations pris depuis le début du $X X^{\mathrm{e}}$ siècle. Des moyens humains et financiers conséquents ont alors été donnés à l'Office national des forêts, anciennement Eaux et Forêts.

${ }^{6}$ Les essences forestières se partagent en deux catégories aux yeux des forestiers : essences objectifs, c'est-à-dire celles qui sont exploitables (chêne, hêtre, dans le cas de Compiègne), et essences non objectifs, toutes les autres, qui seront progressivement éliminées au fur et à mesure de l'exploitation.
} 
fiche descriptive de Prunus serotina, mais dans le contexte américain (Benoit, 1997, p. 22). Dans son aire d'indigénat, $c^{\prime}$ est un arbre très recherché et son bois, de haute valeur, est exporté vers l'Europe. L'agent ne trouve aucun texte mentionnant un risque écologique. Dans ces conditions, bien que Prunus serotina se soit révélé comme un frein aux régénérations, les révisions d'aménagement de 1984 à 2008 le mentionnent sous son appellation vernaculaire erronée de "cerisier à grappes ", et ce en quelques lignes. Il est seulement précisé " qu'il y a lieu de s'efforcer de l'éliminer », sans plus de détails. En 1986, le même agent demande au service des recherches et progrès techniques de l'ONF une expérimentation de sylviculture sur Prunus serotina, pour tenter d'en tirer parti. Cette expérience est un échec. Malgré cela, la révision d'aménagement de 1996 à 2010 (ONF, 1996), qui nomme cette fois l'arbre de façon exacte, reste assez peu prolixe, que ce soit sur l'état des lieux ou les méthodes de lutte. Les conséquences économiques n'ont pas été quantifiées. Le silence relatif sur ce sujet devient compréhensible si l'on sait que l'ONF est assez mal perçu à Compiègne, car accusé de vouloir exploiter exagérément la forêt. Pointer du doigt ce problème, ne serait-ce que pour tenter de le résoudre, reviendrait à souligner des méthodes sylvicoles très controversées, donc raviver un sujet douloureux.

En parallèle, une association ${ }^{7}$ s'intéressant au fonctionnement et à la vie des forêts de la région se rend compte, dans les années 1975-1980, lors d'une action en forêt de Compiègne, de la présence de cet arbre inconnu en très grande quantité. Après information auprès de l'ONF, qui lui apprend les problèmes de régénération, cette association ne prendra aucune mesure contre l'arbre. Les invasions biologiques ne sont toujours pas dans l'air du temps.

$\mathrm{Au}$ niveau international, la question des invasions biologiques est rendue publique en 1992 par la Convention sur la diversité biologique (CDB). L'Union internationale pour la conservation de la nature (UICN) établit ce phénomène comme deuxième cause d'appauvrissement de la biodiversité. Ce thème, en même temps que ceux abordés par la Convention, devient très médiatisé. En France, le programme INVABIO est lancé en 1999 par le ministère en charge de l'environnement, relayé par INVABIO II en 2001.

Retour en Picardie en 1999, lorsqu'un ingénieur agronome et écologue travaillant au conseil régional prend connaissance du problème des invasions biologiques et des conséquences sur la biodiversité. Il demande alors un état des lieux pour la Picardie au Conservatoire

\footnotetext{
${ }^{7}$ La Société des amis des forêts d'Halatte, Ermenonville et Chantilly, que rejoint toute personne intéressée par les interventions de naturalistes, botanistes, éthologues, etc., par des sorties terrain à la découverte du patrimoine faunistique et floristique régional, de même que par toutes les interventions étatiques en lien avec les forêts (construction de routes, plan de chasse, etc.).
}

botanique national de Bailleul (CBNBL). Des plantes sont alors identifiées comme invasives (Hendoux, 1999). En 2001, le conseil régional de Picardie (CRP), le CBNBL et une société savante naturaliste (Société linnéenne Nord-Picardie, SLNP) lancent en conséquence le programme Alerte et lutte contre les espèces invasives en Picardie. Ce programme permet la publication d'une plaquette, Alerte aux plantes invasives, où apparaît Prunus serotina. Elle est distribuée aux bureaux d'études, aux élus, aux associations, à la direction départementale de l'Équipement (DDE) et aux pépiniéristes. Toujours dans le cadre de ce programme, une journée d'information est organisée à Amiens, en 2003, par la SLNP et le CBNBL, de façon à informer les usagers régionaux, notamment par le biais des fédérations.

Quant aux publications, elles illustrent la croissance exponentielle de l'intérêt pour le sujet depuis la Convention sur la diversité biologique. Les premiers textes concernant les invasions biologiques apparaissent donc dans les années 1990. Ils sont de nature soit scientifique, soit professionnelle. Progressivement, le sujet se démocratise et atteint, une dizaine d'années plus tard, les revues grand public de jardinage ${ }^{8}$. Certaines revues équivalentes font cependant le choix de ne pas publier, soit par manque d'intérêt, soit parce que l'équipe de rédaction ne considère pas ce phénomène comme suffisamment spectaculaire et nocif. Quant aux journaux, seuls Le Monde et Libération consacrent une à deux pages au phénomène, en reprenant les arguments énoncés, notamment, par l'UICN ${ }^{9}$. Le type d'informations dans ces revues ou journaux suit le gradient correspondant aux connaissances scientifiques des auteurs. Plus on s'approche du domaine du grand public, plus l'information est partielle et ciblée sur une question pragmatique, tels le risque économique d'une invasion ou ses dangers sanitaires ${ }^{10}$. Enfin, en 2004, une revue dédiée aux professionnels des espaces naturels publie, dans un de ses numéros, un dossier sur les plantes envahissantes ${ }^{11}$. Les articles font le bilan des connaissances scientifiques sur le sujet, les auteurs sont des chercheurs ou des professionnels ayant été confrontés à des cas d'invasion biologique. La démarche est pragmatique, puisqu'un tableau des techniques d'éradication y est présenté. Une coordination

\footnotetext{
8 «Un jardin formidable!», L'Ami des jardins, 28 juillet 2002; "Pourquoi les plantes aquatiques sont-elles aussi chères?", L'Ami des jardins, 12 juin 2003 ; «Jardiniers, attention aux plantes fugueuses! », Rustica, 9 juillet 2003.

9 «La biodiversité menacée par les plantes invasives", Le Monde, 6 novembre 2002; "Alerte aux plantes envahissantes venues de la biosphère », Libération, 5 juin 2001.

10 «Pour comprendre la nature», Le Parisien, 11 novembre 2001 ; «Alerte aux plantes invasives », Le Courrier picard, 17 juin 2003.

11 «Plantes envahissantes, que faire?", Espaces naturels, 5, janvier 2004; numéro téléchargeable sur Internet : http://www.espaces-naturels.fr/site/revue-5.pdf
} 
entre les différents acteurs serait-elle en train de se mettre en place, comme le prônait déjà Muller (2000)?

\section{Les réactions à propos de Prunus serotina à Compiègne : un fossé entre catégories sociales}

Face à cette prise de conscience par un public averti des enjeux des invasions biologiques, argumentée par des études scientifiques, le grand public de Compiègne reste peu réactif sur le sujet. Aucune des différentes catégories sociales identifiées lors des enquêtes n'a montré un intérêt pour la question, hormis celles ayant des connaissances approfondies sur les enjeux écologiques dans le domaine. Certains connaissent le Prunus serotina, tels les exploitants (scieries ou ébénisteries) qui l'utilisent en fonction de leurs besoins et du marché. Certains propriétaires forestiers le connaissent également et parfois cherchent à le planter, les prix de vente de ce bois aux États-Unis les faisant rêver. Les élus ont pu avoir été avertis, mais ils doivent faire face à des besoins qu'ils qualifient de plus urgents, comme le chômage ou la construction de voies de communication. Ils se doivent de répondre aux attentes de la population; or, Prunus serotina n'est pas l'objet, jusqu'à présent, d'un mouvement écologiste, naturaliste, patrimonial ou autre. En outre, ils se réfugient derrière l'absence de procédure existante dans ce genre de situation. «Qui fait quoi?» résument-ils. Marsal (2002) note déjà la même situation d'une manière plus globale. Les élus disent attendre de toute façon les résultats de la recherche sur Prunus serotina avant de réfléchir aux moyens d'agir, de concert avec les associations naturalistes ou autres organismes régionaux professionnels ou grand public. Ces organismes, quant à eux, sont déroutés, notamment par le manque de moyens financiers ou humains à leur disposition, mais également par le manque de coordination dans ce domaine. Ils semblent être malgré tout les plus crédibles, pour reprendre l'expression de Jollivet (1997). Toutes les tentatives ayant eu lieu pour lutter contre des invasifs en Picardie sont indépendantes les unes des autres, non concertées et donc hétéroclites. Il n'existe pas de processus étalonné, donc pas de prise de relais des quelques essais effectués. Ceux-ci sont dus à la volonté d'individus sensibilisés à la question par leur parcours professionnel et personnel. Ils tentent, seuls ou en impliquant un organisme avec lequel ils ont un lien, sur la base de leurs convictions, de mettre en place des luttes contre les espèces invasives. Ils se retrouvent en situation d'experts souhaitant agir pour aider à conserver la biodiversité. À Compiègne, la catégorie des experts se confond dans les actes avec celle des lanceurs d'alerte (Chatauraynaud et Torny, 1999). Ces derniers y sont au nombre de deux : un écologue travaillant au conseil régional et qui est à l'origine du programme décrit précédemment (Alerte et lutte contre les espèces invasives) et un expert forestier privé membre de Prosilva ${ }^{12}$. Ce combat se fait au nom de la biodiversité, du patrimoine, c'est-à-dire de notions associées à un paysage intellectualisé, distancié, que ne partage pas forcément le grand public. On peut également s'interroger sur la place des arguments sociaux et écologiques opposables au «vagabondage » des espèces décrit par Clément (2002).

Parmi ceux qui ne connaissent pas Prunus serotina, dont des randonneurs ou des personnes habitant en forêt, certains l'ont remarqué sans approfondir leur recherche à ce sujet. Ils axent plutôt leurs priorités sur la propreté des chemins ou du sous-bois, ou la persistance des arbres anciens autour de leur habitation. Ils ont cependant inventé des usages en rapport avec cet arbre dont ils apprécient l'esthétique. Certains ont élaboré un parcours de randonnée particulier en automne, de manière à passer dans les zones où il est abondant pour admirer son feuillage doré. D'autres ramassent ses fruits et en font des confitures. Les cavaliers aiment passer là où il se trouve pour manger quelques baies au cours de leur balade. Enfin, des personnes viennent déterrer des plants pour les réinstaller dans leur jardin où ils souhaitent l'admirer. Certains en arrivent même à agresser les agents de l'ONF qu'ils voient en train de couper du Prunus serotina. La présence de cet arbre est donc anecdotique et peut se cantonner à quelques usages périphériques, spécifiques à chaque groupe d'usagers, sans qu'il y ait d'échange entre eux. En témoignent les différentes dénominations de l'arbre selon chaque groupe (merisier à grappes, bois puant, etc.). Cela illustre le peu de connaissances de la population sur le Prunus serotina, contrairement aux essences fédératrices que sont le chêne et le hêtre, qui représentent le patrimoine de la forêt de Compiègne (Callais, 1998). Une éducation serait donc nécessaire s'il y avait une volonté d'éradication. Le regard de ces personnes sur cet arbre n'a pas été formé à le voir en tant qu'envahisseur biologique.

\section{Les invasions biologiques : un problème de médiatisation?}

Il est intéressant de constater la multiplication des identités de Prunus serotina en l'absence d'une opinion fédératrice. Cet exemple en forêt de Compiègne illustre les existences diverses que peut prendre un même objet à partir d'interprétations, c'est-à-dire de visions de la forêt, différentes de la part de chaque catégorie sociale (Picon, 1996; Claeys-Mekdade, 2000). On oscille entre la situation où il devient le centre des préoccupations et celle où il reste invisible (Javelle, 2005). Ce dernier cas s'observe chez certaines personnes qui cherchent en forêt des

\footnotetext{
12 Association de forestiers prônant une gestion des forêts le plus proche possible de l'état «naturel».
} 
éléments répondant à leurs attentes et éliminent littéralement de leur champ de vision toute autre chose. Un tel état de fait est ici créé par la nouveauté de la situation, qui perdure à cause de la « discrétion » de Prunus serotina, n'obligeant donc pas les institutions étatiques ou régionales à intervenir en urgence (Roux, 2003), comme c'est le cas pour la jussie ${ }^{13}$ actuellement en Picardie. On peut d'ailleurs se demander quelles raisons l'emportent dans la décision d'éradiquer une espèce : pour répondre aux demandes de la population? Les griffes de sorcière présentes sur le littoral méditerranéen sont appréciées pour leur esthétique, comme le Prunus serotina, et sont le but de promenades du grand public, mais aussi l'objet d'une campagne d'éradication. Pour l'aspect sylvicole? N'oublions pas qu'au début du XXe siècle, Prunus serotina était vu comme essence précieuse, comme améliorateur de la litière sous résineux et comme pare-feu. Même la prestigieuse Société botanique de France prônait son introduction dans nos forêts. Starfinger et al. (2003) décrivent très bien cette instabilité de statut. Pour l'aspect écologique? Dans les cas du Prunus serotina et de la jussie, les conséquences exactes de leur développement restent encore mal connues. En dehors du fait que l'on constate l'explosion de Prunus serotina depuis environ quarante ans, rien ne permet de dire quelle sera son évolution dans les quarante prochaines années. Des cas plus anciens d'invasion biologique ont montré une régression naturelle de l'espèce au bout d'un certain temps. Par ailleurs, il ne faut pas oublier qu'une partie des espèces animales et végétales considérées actuellement comme autochtones ont été allochtones auparavant (Lambinon, 1997). Dans les deux cas du Prunus serotina et de la jussie, des experts s'attèlent au problème. Dans l'un seulement, ils sont relayés par des élus au vu des conséquences économiques : la jussie a été listée parmi les plantes invasives, et les élus l'ont prise pour cible puisqu'elle freinait le tourisme fluvial par son extension excessive. Outre cet aspect matériel, une volonté d'éradication n'est-elle pas également la conséquence d'une médiatisation souvent moralisante, qui demande de "protéger la nature » au nom des générations futures, de manière à corriger les excès de notre civilisation ? C'est ainsi que certaines espèces deviennent des sortes de bouc émissaire de groupes sociaux. Il faut garder à l'esprit le décalage entre les données initiales indiquant les risques biologiques de la présence de telle ou telle espèce et la lutte effective sélectionnant les espèces selon des intérêts souvent autres qu'écologiques (Laurent, 1990). Loin de minimiser les dangers d'une invasion biologique, ce texte entend faire réfléchir, comme le fait Bruno (1990) d'une manière plus générale, sur l'utilisation des données scientifiques dans ce domaine.

\footnotetext{
${ }^{13}$ Ludwigia grandiflora, une plante aquatique envahissante introduite volontairement d'Amérique du Sud pour ses qualités esthétiques.
}

\section{Pour conclure : forêt et biodiversité, une grammaire en gestation}

Sans mise en ordre du discours, l'invasion biologique n'existe pas en tant que représentation collective, car elle ne peut se révéler aux différents acteurs, ce qui empêche toute possibilité d'anticipation et de prévention du phénomène. Le problème ne peut être au mieux interprété que dans les cadres de connaissance et de pratique usuelle des différents acteurs, qu'ils soient des professionnels, des membres d'associations ou des usagers. Faute de schéma global existant (cognitif, organisationnel et législatif), Prunus serotina n'a pas de visibilité sociale et n'a pu être l'objet d'un processus social d'identification, malgré son premier signalement en 1970 par le responsable régional forestier. Seuls quelques individus ont joué le rôle de passeur de domaines, par exemple du champ scientifique à celui de la vie associative, sans cependant avoir les moyens nécessaires pour prendre en charge le problème à différents niveaux d'organisation (privé, public, associatif, etc.) et d'échelle (locale, nationale).

Les cadres d'intégration restent partiels, sectorisés et inadaptés aux problèmes nouveaux et inédits posés par les invasions biologiques. Il n'y a pas de pensée intégrative en mesure de déboucher sur une véritable stratégie d'action. L'absence d'arrière-plan cognitif entraîne un rabattement vers les savoirs et les procédures routinières d'action. Dans cette perspective, seules des initiatives personnelles sont menées ; mais, malheureusement, coupées de tout réseau institutionnel et de soutien, elles ne peuvent aboutir et s'inscrire durablement. L'efficacité des actions en est alors freinée par l'impossibilité de faire les liens entre les expériences individualisées, étant donné l'absence de transfert, de traduction sociale et donc d'apprentissage. D'où la pauvreté des actions de défense (qui semblent relever plus d'un traité d'alchimie - arrachage à la main, broyage - que de méthodes rationnelles) et le manque de réflexivité et de capacité d'innovation. Les préoccupations des uns et des autres restent motivées par des intérêts propres (Campbell, 1997), sans rapport avec le phénomène et sa compréhension. Chaque catégorie sociale est centrée sur ses préoccupations, sans qu'il $\mathrm{y}$ ait une vision systémique et globale de la situation et des intérêts en jeu autres que corporatistes ou professionnels. De ce fait, Prunus serotina est resté l'affaire de quelques initiés, botanistes et écologues. En résulte une non-reconnaissance, si l'on compare à d'autres invasions (Dalla Bernardina, 2000 ; Dammekens, 2001 ; Adam, 2001) telles que celle de Caulerpa taxifolia ${ }^{14}$ (Friedman, 2001), et une absence de statut social sur les plans tant

\footnotetext{
14 Une algue verte des mers tropicales qui envahit les fonds marins méditerranéens depuis qu'elle y a été rejetée par le Musée océanographique de Monaco, surnommée «l'algue tueuse » pour souligner son effet nocif sur les herbiers de posidonie.
} 
patrimonial (pas d'appropriation) qu'économique (absence de valorisation). Cela ne prédispose pas à la mise sur agenda politique du problème, qui reste circonscrit au local et à un petit cercle d'avertis.

La nature - ici la forêt en tant qu'espace de biodiversité - n'est donc pas une donnée naturelle. Elle est un fait construit, local et national, conséquence à la fois de pratiques de significations discursives, de formes stables de connaissances et d'activités matérielles et institutionnelles (aménagements sylvicoles, modes de traitement et d'exploitation, type de statut : domanial ou privé). Cette posture ethnologique de recherche conduit à déconstruire l'idée d'une séparation ontologique entre nature, science, technique et société, et à envisager la forêt comme le résultat d'un processus dynamique d'interactions entre des hommes et des écosystèmes, associant des composantes biologiques et sociales qui modifient les représentations et l'usage dont les forêts font l'objet. L'ethnographie de l'invasion et de sa relation aux différents acteurs sociaux consiste à examiner comment cette réalité émerge, circule, à observer le phénomène comme un processus dynamique plutôt que comme une structure stable. Dans cette perspective, les scientifiques participent, de même que les autres acteurs (Mougenot, 2003), à la construction sociale de la biodiversité et à sa réalisation comme problème public et aboutissant à sa reconnaissance partagée. La biodiversité n'est pas seulement, comme le montre cette analyse, l'affaire des savants, car il est impossible d'isoler la science de la société, de la cloisonner. Les chercheurs produisent la connaissance non plus dans le confinement étroit de leur cercle, mais dans la confrontation avec l'ensemble des acteurs sociaux, usagers comme gestionnaires. Tel est le sens de ce récit sur les trajectoires scientifiques, techniques, sociales et spatiales de l'intrus Prunus serotina dans la forêt de Compiègne.

\section{Remerciements}

Cette étude a reçu le soutien financier du ministère de l'Écologie et du Développement durable (MEDD) dans le cadre du programme INVABIO II (CR n09-D/2003).

\section{Références}

Adam, H., 2001. Les Invasions naturelles dans la presse ancienne de Provence et de Bretagne, Brest, Université de Bretagne Occidentale.

Benoit, Y., 1997. Le Guide des essences de bois, Paris, CTBA/Eyrolles.

Bertho-Lavenir, C., 1999. La Roue et le stylo: comment nous sommes devenus touristes, Paris, Odile Jacob.

Bruno, J.-B., 1990. La sacralisation de la science, in Rivière, C., Piate, A., Nouvelles idoles, nouveaux cultes, Paris, L'Harmattan.
Callais, F., 1998. À la découverte des forêts de Compiègne, Laigue et Ourscamps-Carlepont, Compiègne, Société historique de Compiègne.

Campbell, F.T., 1997. Exotic pest plant council: cooperating to assess and control invasive non indigenous plant species, in Luken, J.O., Thiriet, J.W. (Eds), Assessment and Management of Plant Invasion, New York, Springer, 228-243.

Chatauraynaud, F., Torny, D., 1999. Les Sombres Précurseurs : une sociologie pragmatique de l'alerte et des risques, Paris, EHESS.

Claeys-Mekdade, C., 2000. Les Conflits d'aménagement : rapport à la nature et rapport sociaux. Thèse de doctorat d'État, Université de Provence, Aix-en-Provence.

Clément, G., 2002. L'Éloge des vagabondes, Paris, Nil Éditions.

Dalla Bernardina, S., 2000. Étude anthropologique comparative sur la prolifération des algues sur les côtes françaises, Brest, Université de Bretagne Occidentale.

Dammekens, S., 2001. Exemple de la prolifération algale sur les côtes bretonnes: l'algue verte, symbole de pollution et facteur de désordre, Brest, Université de Bretagne Occidentale.

Friedman, S., 2001. Représentations vernaculaires de la prolifération algale caulerpa taxifolia dans la région d'Hyeres (Var), Brest, Université de Bretagne Occidentale.

Graves, L., 1983. Précis statistique du canton de Compiègne, arrondissement de Compiègne, Paris, Librairie Guénégaud.

Hendoux, F., 1999. Les Espèces naturalisées ou en voie de naturalisation à caractère de "peste végétale » en Picardie, Bailleul, CBNBL.

Hough, A.F., Forbes, R.D., 1943. The ecology and silvics of forests in the high plateaux of Pennsylvania, Ecological Monographs, 13, 299-320.

Javelle, A., 2005. Plantes envahissantes et perception sociologique, le cas du cerisier tardif à Compiègne, La Garance voyageuse, 70 .

Jollivet, M., 1997. La construction sociale des problèmes d'environnement : pourquoi et comment certains problèmes émergent et d'autres pas?, Nature Sciences Sociétés, 5, 2, 71-76.

Joly, P., 2000. Invasions biologiques : état de l'art et perspectives, Revue d'écologie - Terre Vie, suppl. 7, 21-35.

Kalaora, B., 1993. Le Musée vert, Paris, L'Harmattan.

Kalaora, B., Savoye, A., 1986. La Forêt pacifiée : sylviculture et sociologie au XIX' siècle, Paris, L'Harmattan.

Lambinon, J., 1997. Les Introductions de plantes non indigènes dans l'environnement naturel, Strasbourg, Éditions du Conseil de l'Europe.

Laurent, P.-J., 1990. Point sur les risques engendrés par l'introduction intempestive de l'écrevisse rouge des marais de Louisiane, Le Courrier de l'environnement de l'INRA, 11, 7-10.

Léré, J.A.F., 1819-1834. Traité historique de la Faisanderie et de l'éducation artificielle des faisans, Fonds Léré, bibliothèque Saint-Corneille, Compiègne.

Léré, J.A.F., 1827-1839. Notes sur la botanique et horticulture à Compiègne, Fonds Léré, bibliothèque Saint-Corneille, Compiègne.

Marsal, P., 2002. Les invasions biologiques, Le Courrier de l'environnement de l'INRA, 46, 103-108.

Mougenot, C., 2003. Prendre soin de la nature ordinaire, Paris, INRA Éditions.

Muller, S., 2000. Les espèces végétales invasives en France : bilan des connaissances et propositions d'actions, Revue d'écologie - Terre Vie, suppl. 7, 53-69. 
ONF, 1996, Aménagement forestier, révision d'aménagement, 19962010, Compiègne.

Picon, B, 1996. Du bon usage de la menace : chronique des représentations de la nature en Camargue, Études rurales, 141-142, 143-156.

Roux, J., 2003. Où se loge la collectivité d'un risque collectif? L'exemple de la pollution industrielle des sols, in Gilbert, C. (Ed.), Risques collectifs et situation de crise : apports de la recherche en sciences humaines et sociales, Paris, L'Harmattan.
Starfinger, U., 1997. Introduction and naturalization of Prunus serotina in Central Europe, in Brock, J., Wade, P.M., Pysek, P., Green, D. (Eds), Plant Invasions: Studies from North America and Europe, Leiden, Backhuys Publishers, 161-171.

Starfinger, U., Kowarik, I., Rode, M., Schepker, H., 2003. From desirable ornamental plant to pest to accepted addition to the flora? The perception of an alien tree species through the centuries, Biological Invasions, 5, 323-335.

Theys, J., Kalaora, B., 1998. La Terre outragée, Paris, Latitudes. 